\title{
Is there a cancer knowledge paradox? Risk perception and knowledge of breast and colon cancer in a diverse population
}

\begin{abstract}
Objective: The accuracy of cancer risk perception has implications for preventive behaviors. Data shows women overestimate their numeric risks. Breast cancer's media presence is highly visible, perhaps increasing knowledge but inflating risk perceptions. This study explores relationships between breast cancer (BC) and colon cancer (CC) knowledge and numeric risk perceptions among women. We hypothesize that general knowledge of $\mathrm{BC}$ will be high relative to $\mathrm{CC}$, but risk perception for $\mathrm{BC}$ will be less accurate.
\end{abstract}

Methods: Risk perception and knowledge data was collected from 490 female patients recruited for the Kin Fact study from a Women's Health Clinic. Women completed baseline surveys and $\mathrm{CA}$ Gene software was used to calculate actual lifetime risk for $\mathrm{BC}$ and $\mathrm{CC}$. Correlations, t-tests and linear regressions were used for analysis

Results: Women averaged 33years old, and 59\% were African American. Women overestimated their numeric risk for both, but the mean BC overestimation $(29 \%)$ was significantly larger than CC (19\%). Average knowledge scores for BC were also significantly higher than CC. Compared to knowledge about CC, women who had greater knowledge of $\mathrm{BC}$ also were more inaccurate in terms of their perceived numeric risk $(\mathrm{r}=-0.126, \mathrm{p}=0.006)$. This finding remained significant controlling for age, race and genetic literacy.

Conclusion: Results endorse an apparently paradoxical effect that compared with $\mathrm{CC}$; women with increased knowledge of $\mathrm{BC}$ have less accurate risk perception for $\mathrm{BC}$. Inaccuracies in perceived risk can affect psychosocial well-being and adherence to screening recommendations. Findings reveal need for increasing knowledge without increasing risk misperceptions.
Volume I Issue 2 - 2014

\author{
Katherine A Lang, John M Quillin, Donna \\ McClish, Deborah J Bowen, Maria Gyure, \\ Alexander H Krist, Vivian M Rodriguez, \\ Rosalie Corona, Joseph Borzelleca, Joann N \\ Bodurtha \\ Northside Hospital Cancer Institute, USA
}

Correspondence: Katherine Lang, Northside Hospital Cancer Institute, 1000 Johnson Ferry Rd NE,Atlanta, GA 30342, USA, Tel97857876340, Email katie.lang@northside.com

Received: September 02, 2014 | Published: September 22, 2014

\section{Introduction}

\section{Background}

Breast and colon cancers are the second and third most common cause of cancer death among US women, respectively. ${ }^{1}$ Adherence to mammography and colonoscopy guidelines is therefore a significant aspect of women's healthcare in light of the reduction in mortality associated with proper screening for these cancers. ${ }^{2,3}$ Despite this benefit, many women miss either one or both of these screenings. ${ }^{4-7}$ Additionally concerning is the increasing disparity in survival for these cancers among African-American women. While strides are being made in reducing cancer deaths overall, breast and colon cancer outcome disparities between African-American and Caucasian patients appear to be increasing. ${ }^{8}$

Limited access to healthcare and lack of health insurance are well described obstacles to screening. ${ }^{9-12}$ While public health programs and major federal legislation have been developed to address this disparity, socioeconomic factors alone do not completely account for the deficiency in recommended cancer screening. Internal and personal barriers to adherence including fear, lack of knowledge, misperceptions of cancer risk and cultural norms all inform a complex and highly individualized decision-making process. ${ }^{7,13}$ Cancer risk perception is one internal element that is a significant contributing factor to screening decisions and health behaviors. ${ }^{14-18}$ The formation of risk perception is a complex psychological process that incorporates many facets including numeracy, emotional factors and knowledge. ${ }^{14}$
Generalizable conclusions about the development of individual cancer risk perception have been difficult to elucidate, however one consistent finding of this literature is that women overestimate their risk for breast cancer, particularly when estimating numeric risk. ${ }^{19}$ A possible contribution to this over estimation is the ubiquitous messaging of various breast cancer awareness and fundraising campaigns. Research into this presence found a significant overrepresentation of breast cancer-centered articles in popular magazines when compared to cardiovascular disease articles, despite the higher mortality rate for women with heart disease. This discrepancy in coverage was found to be increasing over time. ${ }^{20}$ Additional research has found that one group of women who may be particularly susceptible to this messaging, those with a family history of breast cancer end to underestimate their risk for other conditions, including heart disease and colon cancer. ${ }^{21}$

Other research shows that women with a family history of breast cancer, or those with benign breast disease tend to overestimate their breast cancer risk when compared to women without these factors, which is evidence of the availability heuristic at work. ${ }^{22-24}$ The availability heuristic is the idea that a person judges the likelihood of an event or outcome based on how easily an example of that outcome can be conceived.$^{25}$ Risk overestimations could also be attributed to a bias in thinking where perceptions are based on emotion rather than analytical reasoning. ${ }^{26}$ Clearly there is a delicate and difficult balance between informing women of common health risks and population based recommendations while not skewing individual perceptions towards one specific disease. 
Not all cancers have received the same widespread attention and messaging as breast cancer. A study of adults' understanding of colon cancer screening found significant deficiencies. The Health Information National Trends Study (HINTS) surveyed over 3,000 US adults and found that only $40 \%$ knew the recommended age to begin colonoscopies and even less, $(13 \%)$ knew the proper frequency of screening. This lack of knowledge was associated with younger ages, less education and race, specifically, African American. ${ }^{27}$ Prior studies of screening barriers often assess risk perception or knowledge; however few assess both aspects in a single research question. It is important to understand this relationship as each of these factors contributes to women's overall screening choices. Therefore, this study aims to investigate the relationship between women's knowledge of both breast and colon cancers as well as the accuracy of personal risk perception for these cancers. In order to assess this research question, we analyzed the accuracy of women's numeric risk perception and their cancer knowledge for breast and colon cancer. We then tested whether discrepancies in perceived risk were correlated with discrepancies in knowledge and if race, ethnicity or genetic literacy impacted this relationship.

\section{Methods}

This study was a secondary analysis of survey data from the KinFact, (Keeping Information about Family Cancer Tune-up) study (R01 CA140959), a randomized controlled trial examining the effects of an intervention on family communication about hereditary cancer. Only baseline data from KinFact obtained prior to the study intervention was analyzed for this cross-sectional study.

\section{Participant recruitment and baseline survey}

Study participants were recruited from two urban women's health clinics in Richmond, Virginia. A convenience sampling method was used in two clinic waiting rooms. All English-speaking women 18 years of age or older who were competent to give consent and were patients at the clinic were eligible for enrollment. Recruitment began in July 2010 and was completed in January 2012, and 490 total women were randomized.

At the time of recruitment, participants completed a baseline survey that included questions on family communication, ${ }^{28}$ as well as risk perception and knowledge measures. They were then randomized into intervention and control groups. The intervention was a 20minute tailored discussion based in the coordinated management of meaning for improving family communication and modeled after the 6-step process adapted by Daly ${ }^{29}$ from Buckman ${ }^{30}$ "breaking bad news" technique. ${ }^{28-30}$ This discussion also included a personalized cancer risk assessment (based on reported family history) and riskreduction education. Because this analysis uses data collected prior to randomization, we are not measuring the effects of the intervention on our sample.

\section{Knowledge measure and variables}

Knowledge of cancer information was measured using the Breast Cancer and Heredity Knowledge scale developed by Ondrusek et al., ${ }^{31}$ This scale was designed to assess general knowledge of breast cancer in women with low to moderate risk for hereditary breast cancer. Response options were rated using a 5 point scale,

$$
\begin{aligned}
& \text { a. Totally Agree } \\
& \text { b. Agree }
\end{aligned}
$$

\section{c. Don't Know}

d. Disagree

\section{e. Totally Disagree}

Items answered correctly as either "Totally Agree" or "Totally Disagree" was assigned a score with a value of 2 . Items answered correctly as either "Agree" or "Disagree" were assigned a score with a value of 1 . Responses of "Don't Know" were assigned a score of 0 . Items answered incorrectly with "Totally Agree or "Totally Disagree" were assigned a score of -2 , and items incorrectly answered with "Agree" or Disagree" were assigned -1. Item scores were summed and divided by the total number of questions. Higher scores indicated greater knowledge.

Colon cancer knowledge was measured by adapting the BCHK scale to items to ask about colon cancer. Two of the original items from the 11 item BCHK were excluded due to their specificity to breast cancer. These items were "Swelling or enlargement of one breast may be a sign of breast cancer" and "Men cannot inherit breast cancer gene mutations." Remaining items were tailored to correspond to colon cancer. For example, the original item "Ovarian cancer and breast cancer in the same family can be a sign of hereditary breast cancer" was changed to "Uterine cancer and colon cancer in the same family can be a sign of hereditary colon cancer." Response options were rated using the same scale previously described for the breast cancer knowledge measure.

\section{Risk perception measures and variables}

In order to measure risk perception accuracy, each participants' lifetime risk for breast and colon cancers was determined. During recruitment, a three generation family history was collected by recruiters and entered as pedigrees utilizing CAGene v5.1, a computer interface tool that utilizes several cancer models (Claus, Gail, BRCApro, and MMRpro) to generate the likelihood that an individual carries a germline mutation in a cancer predisposition and provides lifetime risk estimates for breast and colon cancers. For women with no family history of breast cancer, the BRCApro calculation was used for the "calculated risk" variable. For women with a reported family history of breast cancer, the average of the BRCApro and Claus Models were used as the "calculated risk" variable.

The perceived risk variable was obtained from two questions within the baseline survey which asked: "If there are 100 women who are your same age and race/ethnicity, who all have your same risk for developing breast cancer, how many of them do you think will develop breast cancer by the time they are 90years old?" and "If there are 100 women who are your same age and race/ethnicity, who all have your same risk for developing colon cancer, how many of them do you think will develop colon cancer by the time they are 90years old?"Participants' numeric response to this question was the "perceived risk" variable analyzed.

Using these two variables, the variable representing the accuracy of perceived risk was determined by subtracting each participants' perceived risk (obtained from their answers on the baseline survey) and their calculated risk (from their reported family history). We then took the absolute value of this number because the aim was to measure accuracy rather than over or underestimation of risk. Using this method, a smaller overall value in this risk accuracy variable would represent a more accurate risk perception and a larger value represents a less accurate risk perception. This was completed for breast and colon cancer risks. 


\section{Final risk perception and knowledge variables}

To compare risk perception of colon cancer with breast cancer, we subtracted the colon cancer risk discrepancy (absolute value) from the breast cancer risk discrepancy (absolute value). This method was chosen because we hypothesized that there would be a larger discrepancy in actual and perceived risk for breast cancer. The absolute value was not taken for this final variable. Therefore, a positive value for the final risk accuracy would represent a more inaccurate breast cancer risk perception, while a negative final value would represent a more inaccurate colon cancer risk perception. Using the two scores from each knowledge measure, a variable was created representing the difference in these two scores by subtracting the colon cancer score from the breast cancer score. A lower value from this variable represented a smaller discrepancy in knowledge scores (i.e. similar knowledge), where a higher, positive, value represented a greater discrepancy (higher breast cancer knowledge). Figure 1 outlines and summarizes the variables analyzed.

\section{Genetic literacy measure}

Genetic literacy was assessed for all participants at baseline using the Rapid Estimate of Adult Literacy in Genetics (REAL-G) measure. REAL-G is an 8 -item measure yielding a score of 0 through 8 that was developed based on the REALM validated measure of health literacy. Scores of 3 or less indicate reading at or below a sixth grade level. ${ }^{32}$

\section{Analysis}

We utilized SAS 9.2 for these analyses. Our first step was to test the difference between participants' knowledge of breast and colon cancer. This was done using a paired t-test. We assessed the accuracies between perceived numeric risk and calculated lifetime risks for each individual, for both breast and colon cancers, using a paired t-test. Linear regression was used to test for modifying effects of age, race, and genetic literacy on the differences in risk perception and knowledge. The final step was to test whether discrepancies in risk accuracy were correlated with discrepancies in knowledge. The correlation analysis was to determine if an inverse correlation existed between risk discrepancies and knowledge discrepancies. Regressions were used to determine if the correlations were affected by age, race or genetic literacy.

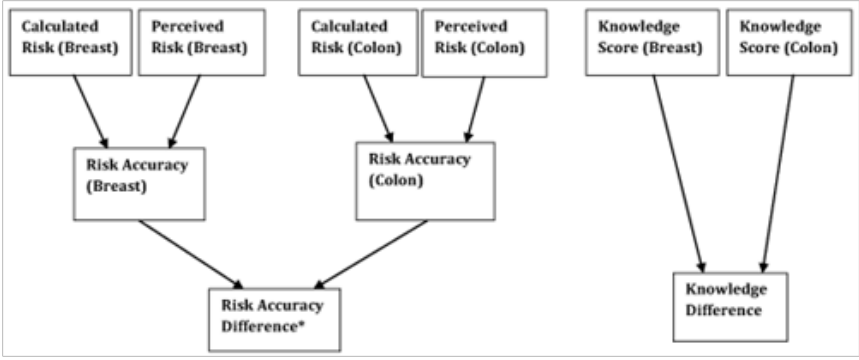

Figure I Risk and knowledge variables.

*Positive value represents more inaccurate breast cancer risk perception; negative value represents more inaccurate colon cancer risk perception

\section{Results}

\section{Participant characteristics}

All participants were female between 18 and 78 years old. Average age was 33years old and almost half (49\%) of participants were between 20 and 29. Four percent of participants were between 18 and 20 . About $14 \%$ of participants were $40-49,8.5 \%$ were $50-59,2 \%$ were
$60-69$, and $0.3 \%$ was over 70 . Overall, $59 \%$ of participants reported their race as African-American, while 33.3\% reported their race as Caucasian. The remaining $7.6 \%$ percent of participants reported a race other than Caucasian or African-American which included those who indicated more than one race, as well as participants of Asian, Native American and Pacific Islander descent. REAL-G measure of genetic literacy showed $17 \%$ of participants tested at a reading level of sixth grade or less.

\section{Risk perception and knowledge findings}

The cancer knowledge measures demonstrated adequate internal consistency (Cronbach's alpha was 0.61 for breast cancer and 0.66 for colon cancer). The maximum and minimum possible knowledge scores were +2 and -2 , respectively. The mean score for breast cancer knowledge was 0.23 , and for colon cancer was 0.14 . The difference between these scores was significant $(\mathrm{p}<0.0001)$. Table 1 summarizes knowledge findings.

Table I Knowledge measure scores

\begin{tabular}{llll}
\hline & N & Mean & Std. Dev. \\
\hline Breast Cancer & 490 & 0.23 & 0.266 \\
Colon Cancer & 490 & 0.14 & 0.252 \\
Difference in Average Score & 490 & 0.09 & 0.264 \\
\hline
\end{tabular}

Table 2 Calculated and perceived cancer risk findings

\begin{tabular}{llllll}
\hline & N & $\begin{array}{l}\text { Mean } \\
(\%)\end{array}$ & $\begin{array}{l}\text { Std. } \\
\text { Dev. }\end{array}$ & $\begin{array}{l}\text { Minimum } \\
(\%)\end{array}$ & $\begin{array}{l}\text { Maximum } \\
(\%)\end{array}$ \\
\hline Colon Cancer & & & & & 14.2 \\
Calculated Risk & 490 & 3.3 & 0.99 & 0 & 90 \\
Perceived Risk & 475 & 21 & 18.5 & 0 & 86.9 \\
Difference & 475 & 18.5 & 17.6 & 0 & \\
Breast Cancer & & & & & 28.5 \\
Calculated Risk & 490 & 10.5 & 2.3 & 0 & 100 \\
Perceived Risk & 475 & 31.7 & 23.7 & 0 & 91.5 \\
Difference & 475 & 24 & 20.4 & 0 & \\
\hline
\end{tabular}

Average calculated lifetime risk was $10.5 \%$ for breast cancer and $3.3 \%$ for colon. The average perceived risk for breast cancer was $31.7 \%$, and for colon cancer was $21.0 \%$. The difference between calculated and perceived risk for both cancers could only be calculated for 475 participants (out of 490) because 15 answers were missing for the perceived numeric risk questions for one or both type of cancer. Table 2 summarizes risk findings.

The average discrepancy between perceived risk and calculated risk was 24.0 for breast cancer and 18.5 for colon cancer. Breast cancer discrepancies were significantly higher than colon cancer discrepancies $(p<0.001)$. The mean overall difference between breast and colon cancer risk discrepancies was +5.4 indicating a greater in accuracy of numeric breast cancer risk perception compared to colon cancer risk perception $(p<0.001)$. Linear regression was utilized to analyze whether race, age, and genetic literacy were predictors of knowledge and/or risk perception discrepancies and none of these relationships reached significance. The final step in analysis was to determine if the discrepancies in risk accuracy were correlated with knowledge. An inverse correlation was found $(\mathrm{p}=0.006 ; \mathrm{r}=-0.126)$ indicating that as knowledge disparities increase (higher knowledge for breast), inaccuracies in perceived risk also increase (greater inaccuracy for breast when compared to colon). Race, age, and genetic literacy were not predictors of knowledge and/or perceived risk correlations. 


\section{Conclusion}

This study examined the understanding of breast and colon cancer risks in a young, majority African-American cohort of women and assessed how risk misperceptions related to their knowledge of these cancers. The results show a potential cancer knowledge paradox. Greater knowledge of breast cancer was correlated with more inaccurate risk perception compared to colon cancer. While, having less knowledge of colon cancer, was correlated with a more accurate risk perception when compared to breast cancer. These results held across all race, genetic literacy and participant ages. About 1 in 5 participants had at or below a sixth grade reading level (based on REAL-G results). Other research shows a negative correlation between self-reported knowledge and actual knowledge in low-literacy women. Women in one such study reported sufficient knowledge, but when their knowledge was measured, their scores indicated low levels. ${ }^{33}$ This is a vital lesson as the patients who need information the most may be least likely to ask for it. Additionally, there are many routes through which a patient may attain knowledge about cancer including media reports, personal experience and education through a clinician. This study could not address whether certain types of information, like media reports, exacerbate inaccurate risk perceptions, while others, like clinician information may not. Further research into this area would be invaluable.

These results contribute to the overall literature on risk perception and knowledge. Adequate cancer knowledge is associated with proper screening adherence. ${ }^{34}$ However, an overestimation of cancer risk can also lead to anxiety and unnecessary use of healthcare resources. ${ }^{35-37}$ When perceived risk is greatly overestimated, particularly for breast cancer, women may be less likely to pursue screening indicating the adoption of a fatalistic view. ${ }^{38}$ Fatalism, or the idea that a cancer diagnosis is inevitable, has been negatively associated with weekly exercise, smoking cessation and proper diet, raising the possibility that an adoption of this belief may indicate higher cancer risks due to poor health behaviors. ${ }^{38}$ Additional studies into fatalism have found that African American women who hold more fatalistic attitudes less likely to follow breast cancer screening guidelines. A study by Spurlock and Cullins ${ }^{39}$ showed an inverse relationship between breast cancer fatalism and age where younger African American women received higher fatalism scores which is particularly concerning as there a decrease in survival for young (under 45) African American women diagnosed with breast cancer. ${ }^{8}$

\section{Limitations}

As with any study relying on participant information, our risk analysis is only as reliable as the history provided by the participants. Therefore, inaccurate family histories may have impacted our estimate of "calculated risk". Additionally, the only question on the baseline survey that assessed numeric risk was a lengthy question that may have been a confusing question for participants. Participants answering this question were asked to give a lifetime risk out of 100 , and while this can easily be converted into a percentage, participants weren't explicitly asked to give their estimated lifetime risk of breast cancer as a probability but rather as a natural frequency. Participants may have answered differently if directly asked to give their estimated lifetime risk as a percentage. Additionally, there is data to suggest that natural frequency may be a more accurate way to elicit numeric information, however this theory could not be assessed using the data available from this study. ${ }^{40,41}$

We attempted to minimize measurement error by using only validated measures in this study. The same knowledge scale was used to measure breast and colon cancer knowledge, although it was only validated for use with breast cancer. At the time that the KinFact study was being designed, there was not a widely accepted and validated colon cancer knowledge measure. Therefore, the BCHK was modified to assess this knowledge. Additionally, two questions were removed from the colon cancer measure which may have impacted our results. Because the study recruitment was done on a volunteer basis in a doctor's office waiting room, there is the possibility of a selection bias as these participants were actively seeking healthcare services, therefore they may be less representative of the population as a whole. Additionally, other potential unmeasured confounders that were not addressed in this study could have impacted the results, including comparative risk perception, insurance status and education.

\section{Future research}

This analysis challenges the assumption that an individual with an appropriate risk perception is necessarily a well-informed patient, or that a patient with sufficient and accurate knowledge also has a realistic risk perception. Additionally, the provision of information may result in an exacerbation of risk misperceptions, especially for breast cancer. While there are many complicated factors that contribute to decreased survival for African American patients diagnosed with breast and colon cancer, this study highlights one potential factor that may be associated with the increased views of fatalism in that population. Our findings were consistent across all races, ages and levels of genetic literacy, indicating that this paradoxical relationship cannot only be attributed to any specific demographic. However, for some women these misperceptions may be contributing to missed screening opportunities.

If further research confirms this paradoxical finding that more knowledge may inflate cancer risk perceptions, particularly for breast cancer and in underserved populations, clinicians could explore ways to gage the perceived risk of their patients. Some options may include a discussion of general cancer risk factors and population based risk estimates. For example, an explanation that most women do not develop breast cancer, however because mammograms have been determined to be a safe and effective screening option, they are recommended for all women once they reach a certain age. Also, a comprehensive discussion of women's health risks which includes heart disease, diabetes and cancer may help to provide perspective on those conditions for which their risk is actually greater. Additionally, when a large amount of publicity is given to some public figures, such as the announcement by Angelina Jolie that she took risk-reducing measures after learning she inherited a BRCA1 mutation, clinicians could consider addressing such events with their patients so they understand that such measures are reserved for women at greatly increased risk and the vast majority of women do not fall into this category.

In the short term, assessing perceptions of risk, understanding of cancer risk factors, and recommendations could help young women appropriately understand their personal cancer risks and may serve as one way to reduce barriers to screening and positively impact cancer outcomes in the long term.

\section{Acknowledgments}

None.

\section{Conflicts of interest}

Authors declare there are no conflicts of interest. 


\section{References}

1. White MC, Espey DK, Kaur JS. United States Cancer Statistics: 19992009 Incidence and Mortality Web-based Report. Department of Health and Human Services, Centers for Disease Control and Prevention, and National Cancer Institute: Atlanta (GA); 2013.

2. Amri R, Bordeianou LG, Sylla P, et al. Impact of screening colonoscopy on outcomes in colon cancer surgery. JAMA Surg. 2013;148(8):747-754.

3. U.S. Preventive Services Task Force. Screening for breast cancer: U.S. Preventive Services Task Force Recommendation Statement. Ann Intern Med. 2009;151(10):716-726.

4. McCaul KD, Tulloch HE. Cancer screening decisions. J Natl Cancer Inst Monogr. 1999;25:52-58.

5. Meadows ES, Whangbo A, McQuarrie N, et al. Compliance with mammography and bone mineral density screening in women at least 50 years old. Menopause. 2011;18(7):794-801.

6. Clarke TC, Soler Vila H, Fleming LE, et al. Trends in Adherence to Recommended Cancer Screening: The US Population and Working Cancer Survivors. Frontiers in Oncology. 2012;27(2):190.

7. Hoffman RM, Lewis CL, Pignone MP, et al. Decision-making processes for breast, colorectal, and prostate cancer screening: the DECISIONS survey. Medical Decision Making. 2010;30(5 Suppl):53S-64S.

8. DeSantis C, Naishadham D, Jemal A. Cancer statistics for African Americans. CA: A Cancer Journal for Clinicians. 2013;63(3):151-166.

9. Hsia J, Kemper E, Kiefe C, et al. The importance of health insurance as a determinant of cancer screening: evidence from the Women's Health Initiative. Preventative Medicine. 2000;31(3):261-270.

10. Catz DS, Green NS, Tobin JN, et al. Attitudes about genetics in underserved, culturally diverse populations. Community Genet. 2005;8(3):161-172.

11. Manswell Butty JA, Richardson F, Mouton CP, et al. Evaluation findings from genetics and family health history community-based workshops for African-Americans. J Community Genet. 2012;3(1):1-12.

12. Orom H, Kiviniemi MT, Underwood W, et al. Perceived cancer risk: why is it lower among nonwhites than whites? USA, Cancer Epidemiol Biomarkers Prev. 2010;19:746-754.

13. Womeodu RJ, Bailey JE. Barriers to Cancer Screening. USA, Medical Clinics of North America. 1996;80(1):115-133.

14. Klein WM, Stefanek ME. Cancer Risk Elicitation and Communication: Lessons from the Psychology of Risk Perception. CA Cancer Journal for Clinicians. 2007;57(3):147-167.

15. Hughes C Lerman C, Lustbader E. Ethnic differences in risk perception among women at increased risk for breast cancer. Breast Cancer Res Treat. 1996;40(1):25-35.

16. Honda K, Neugut AI. Associations between perceived cancer risk and established risk factors in a national community sample. Cancer Detect Prev. 2004;28(1):1-7.

17. Haas JS, Kaplan CP, Des Jarlais G, et al. Perceived risk of breast cancer among women at average and increased risk. $J$ Womens Health. 2005;14(9):845-851.

18. Griffith KA, McGuire K, Royak-Schaler R, et al. Influence of family history and preventive health behaviors on colorectal cancer screening in African Americans. Cancer. 2008;113(2):276-285.

19. Lipkus IM, Kuchibhatla M, McBride CM, et al. Relationships among breast cancer perceived absolute risk, comparative risk, and worries. Cancer Epidemiol Biomarkers Prev. 2000;9(9):973-975

20. Blanchard D, Erblich J, Montgomery GH, et al. Read all about it: the over-representation of breast cancer in popular magazines. Preventative Medicine. 2002;35(4):343-348.
21. Erblich J, Bovbjerg DH, Norman C, et al. It won't happen to me: lower perception of heart disease risk among women with family histories of breast cancer. Preventative Medicine. 2000;31(6):714-721.

22. Tversky A, Kahneman D. Judgment under uncertainty: Heuristics and biases. Science. 1974;185(4157):1124-1131.

23. Kahneman D. A perspective on judgment and choice: mapping bounded rationality. American Psychologist. 2003;58(9):697-720.

24. Facione NC. Perceived risk of breast cancer: influence of heuristic thinking. Cancer Pract. 2002;10(5):256-262.

25. Tversky A, Kahneman D. Availability: a heuristic for judging frequency and probability. Cognitive Psychology. 1973;5(2):207-232.

26. Slovic P, Peters E, Finucane ML, et al. Affect, risk, and decision making. Health Psychol. 2005;24 (4 Suppl):S35-S40.

27. Ford JS, Coups EJ, Hay JL. Knowledge of colon cancer screening in a national probability sample in the United States. Journal of Health Communication. 2006;11(1):19-35.

28. Cronen VE, Pearcea WB, Harrisb LM. The logic of the coordinated management of meaning: A rules-based-approach to the first course in interpersonal communication. Communication Education. 1979;28(1):22-38.

29. Daly MB, Barsevick A, Miller SM, et al. Communicating genetic test results to the family: a six-step, skills-building strategy.Family and Community Health. 2001;24(3):13-26.

30. Buckman R. How to break bad news, a guide for health care professionals. The Johns Hopkins University Press, Blatimore, USA. 1992.pp.240.

31. Ondrusek N, Warner E, Goel V. Development of a knowledge scale about breast cancer and heredity (BHCK). BreastCancer Research and Treatment. 1999;53:69-75.

32. Erby LH, Roter D, Larson S, et al. The rapid estimate of adult literacy in genetics (REAL-G): A means to assess literacy deficits in the context of genetics. Am J Med Genet A. 2008;146A(2):174-181.

33. Davis TC, Arnold C, Berkel HJ, et al. Knowledge and attitude on screening mammography among low-literate, low-income women. Cancer. 1996;78:1912-1920.

34. Graves KD, Huerta E, Cullen J, et al. Perceived risk of breast cancer among Latinas attending community clinics: risk comprehension and relationship with mammography adherence. Cancer Causes Control. 2008;19(10):1373-1382.

35. Quillin J, Fries E, McClish D, et al. Gail model risk assessment and risk perceptions. Journal of Behavioral Medicine. 2004;27(2):205-214.

36. Caruso A, Vigna C, Marozzo B, et al. Subjective versus objective risk in genetic counseling for hereditary breast and/or ovarian cancers. Journal of Experimental and Clinical Cancer Research. 2009;28:157-168.

37. Hopwood P. Breast cancer risk perception: what do we know and understand? Breast Cancer Research. 2000;2(6):387-391.

38. Niederdeppe J, Levy AG. Fatalistic beliefs about cancer prevention and three prevention behaviors. Cancer Epidemiological Biomarkers Prev. 2007;16(5):998-1003.

39. Spurlock W, Cullins LS. Cancer Fatalism and Breast Cancer Screening in African American Women. The ABNF Journal Jan/Feb. 2006;17(1):38-43

40. Cuite C, Weinstein ND, Emmons K, et al. A test of numeric formats for communicating risk probabilities. Medical Decision Making. 2008;28(3):377-384.

41. Cosmides L, Tooby J. Are humans good intuitive statisticians after all? Rethinking some conclusions from the literature on judgment under uncertainty. Cognition. 1996;58(1):1-73. 